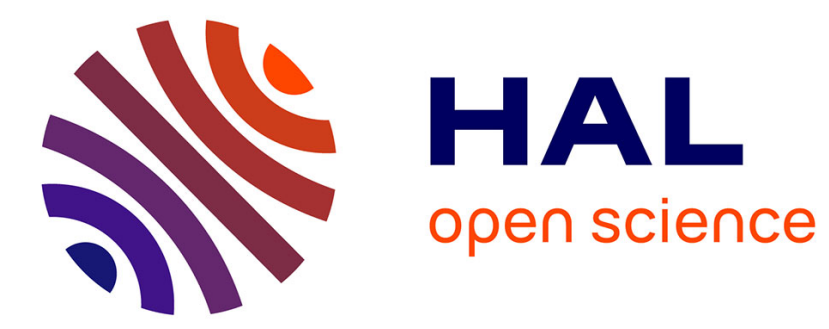

\title{
Groupes fins
}

Cédric Milliet

\section{- To cite this version:}

Cédric Milliet. Groupes fins. The Journal of Symbolic Logic, 2014, 79 (4), pp.1120-1132. 10.1017/jsl.2014.12 . hal-00655411

\section{HAL Id: hal-00655411 \\ https://hal.science/hal-00655411}

Submitted on 28 Dec 2011

HAL is a multi-disciplinary open access archive for the deposit and dissemination of scientific research documents, whether they are published or not. The documents may come from teaching and research institutions in France or abroad, or from public or private research centers.
L'archive ouverte pluridisciplinaire HAL, est destinée au dépôt et à la diffusion de documents scientifiques de niveau recherche, publiés ou non, émanant des établissements d'enseignement et de recherche français ou étrangers, des laboratoires publics ou privés. 


\title{
GROUPES FINS
}

\author{
CÉDRIC MILLIET
}

\begin{abstract}
We investigate some common points between stable and weakly small structures and define a structure $M$ to be "fine" if the topological space $S_{\phi}\left(d c l^{e q}(A)\right)$ has an ordinal Cantor-Bendixson rank for every formula $\phi$ and finite subset $A$ of $M$. By definition, a theory is fine if every of its models is so. Weakly minimal, small, and stable structures are all examples of fine structures. For any of its finite subset $A$, a fine structure has local descending chain conditions on the algebraic closure $\operatorname{acl}(A)$ of $A$ for subgroups uniformly definable over $\operatorname{acl}(A)$. An infinite field with fine theory has no additive or multiplicative proper subgroup of finite index, and no Artin-Schreier extension.
\end{abstract}

\begin{abstract}
RÉsumé. Pour réunir à la fois les structures stables et menues, nous introduisons les structures fines et mettons en évidence plusieurs caractéristiques communes aux structures (faiblement) minimales, stables ou menues : une condition de chaîne uniforme et locale et une notion de presque $\phi$-stabilisateur local. Nous en déduissons qu'un corps infini dont la théorie est fine n'a pas de sous-groupes additifs ni multiplicatifs d'indice fini, ni d'extension d'ArtinSchreier.
\end{abstract}

Les groupes dont la théorie est à la fois stable et menue ${ }^{1}$ ont été étudiés dans $[14,15$, Wagner] où il est montré qu'ils héritent de bien des propriétés des groupes omegastables. L'étude des groupes menus sans hypothèse de stabilité supplémentaire est plus récente $[16,5,7,8]$.

En fait, les structures algébriques menues et celles stables ont de nombreux caractères sinon semblables, du moins analogues. Les corps infinis tout d'abord; qu'ils soient stables ou menus, ils n'ont pas de sous-groupes définissables d'indice fini, ni additifs, ni multiplicatifs [10, Poizat] [9, Milliet]. Les corps menus infinis sont algébriquement clos [16, Wagner], tandis que les corps stables infinis n'ont pas d'extensions d'Artin-Schreier [13, Scanlon], et on conjecture qu'il sont séparablement clos. Les corps gauches de caractéristique positive ayant une théorie menue sont commutatifs [5], et ceux dont la théorie est stable sont de dimension finie sur leur centre [6]. Dans une structure stable, les groupes infiniment définissables sont l'intersection de groupes définissables [3, Hrushovski]. Dans une structure menue, c'est aussi vrai pour les groupes infiniment définissables avec un nombre fini de paramètres [7]. Les groupes stables sont soumis à des conditions de chaîne descendante

2000 Mathematics Subject Classification. 03C45, 03C60.

Key words and phrases. théorie des modèles, rang de Cantor-Bendixson, condition de chaîne locale, extension d'Artin-Schreier.

Merci à Poizat pour ses commentaires élcairés et éclairants.

1. Le terme menu ont est introduit dans [11, Poizat Wagner] comme une traduction infidèle mais bien moins pâle que l'original du mot anglais small (sic) 
uniformes [10, Baldwin Saxl], tandis que les groupes menus sont eux soumis à des conditions de chaîne descendante locales [8]. Les groupes menus ou superstables, s'ils sont infinis, ont un sous-groupe abélien infini [1, Berlin Lascar] [8]. La question est ouverte pour les groupes stables.

Nous essayons ici de pousser un peu plus loin cette analogie et de mettre le doigt sur quelques points communs à ces deux classes de structures. Lorsque cela nous semble possible, nous tentons même de nous passer du théorème de compacité pour englober les structures minimales.

Comme il s'agit de généraliser à la fois la stabilité et le caractère menu, il nous faut à la fois considérer des types locaux (ie des $\phi$-types) et des formules $\emptyset$-invariantes (ie définissables sur le vide). Nous voudrions aussi préserver cette notion en quotientant par une relation d'équivalence définissable, et en ajoutant un nombre fini de paramètres au langage. Plusieurs possibilités, en commençant par la moins restrictive :

(1) pour tout ensemble fini de paramètres $A$, considérer les $\phi$-types sur la clôture définissable de $A$. Cela suffit pour démontrer 8 et 9 , et pour obtenir des conditions de chaîne locales sur $\operatorname{dcl}(A)$, mais pas sur $\operatorname{acl}(A)$;

(2) pour tout ensemble fini $A$, considérer les $\phi$-types sur la clôture algébrique de $A$ formés de formules $A$-invariantes. Cela suffit à démontrer tous les résultats présentés, mais ça n'est pas préservé par interprétation;

(3) pour tout ensemble dénombrable $A$, et tout sous-ensemble fini $B$, considérer les types sur $A$ formés de $\phi$-formules $B$-invariantes;

(4) pour tout ensemble fini de paramètres $A$, considérer les $\phi$-types sur la clôture imaginaire $d c l^{e q}(A)$ de $A$. Cette condition est plus forte que 2, mais sûrement plus naturelle.

\section{NotATIONS}

$L$ est un langage, $M$ une $L$-structure, $\mathfrak{C}$ une extension saturée de $M$ et $A$ un ensemble de paramètres.

On note $A u t(\mathfrak{C} / A)$ le groupe des $L$-automorphismes fixant $A$ point par point. La clôture définissable de $A$ notée $d c l(A)$ est l'ensemble des points de $M$ qui sont invariants sous l'action de $\operatorname{Aut}(\mathfrak{C} / A)$. La clôture algébrique de $A$ notée $\operatorname{acl}(A)$ est l'ensemble des points de $M$ qui ont une orbite finie sous cette action. Si $n$ est un entier naturel, $E$ une relation d'équivalence sur $M^{n}$ définissable sur $A$, on note $a_{E}$ la classe d'équivalence d'un point $a$ de $M^{n}$ et on dit que $a_{E}$ est un paramètre imaginaire (typiquement, dans un groupe, un coset d'un sous-groupe $A$-définissable). L'élément $a_{E}$ est dans la clôture imaginaire de $A$ s'il est fixe sous l'action naturelle de $A u t(\mathfrak{C} / A)$ sur $M^{n} / E$. On note $d c l^{e q}(A)$ l'ensemble des éléments de la clôture imaginaire de $A$. Si $\phi(x, a)$ est une formule et $a$ un uplet de $M^{n}$, par définition, $\phi\left(x, a_{E}\right)$ est la formule $(\exists b)(a E b \wedge \phi(x, b))$.

Etant donnée une formule $\phi(x, y)$ dans $L$, on appelle $\phi$-formule sur $A$ toute disjonction finie de conjonctions finies de formules du type $\phi(x, a)$ ou $\neg \phi(x, a)$ où $a$ est un uplet de paramètre variant dans $A$. Nous disons qu'une $\phi$-formule est $A$-invariante si elle est définissable à paramètres dans $A$, ou de manière équivalente si l'ensemble $\phi(\mathfrak{C}, a)$ des réalisations de $\phi(x, a)$ est globalement invariant sous l'action de $\operatorname{Aut}(\mathfrak{C} / A)$. Insistons sur le fait que si une $\phi$-formule sur $A$ est bien sûr $A$-invariante, 
une $\phi$-formule $A$-invariante n'est pas toujours equivalente à une $\phi$-formule sur $A$ (dans le langage des corps, prendre par exemple pour $\phi(x, y)$ la formule " $x=y "$ : la formule " $x=i \vee x=-i$ " est une $\phi$-formule $\emptyset$-invariante, mais n'est pas une $\phi$ formule sur le vide). Un $\phi$-ensemble sur $A$ est un ensembe défini par une $\phi$-formule sur $A$.

Un $\phi$-type sur $A$, c'est un ensemble consistant de $\phi$-formules sur $A$. On note $t p_{\phi, A}(x)$ l'ensemble des $\phi$-formules sur $A$ vérifiées par $x$. C'est un $\phi$-type complet sur $A$. On note $S_{\phi}^{M}(A)$, ou simplement $S_{\phi}(A)$ si $M$ n'est pas ambiguë, l'ensemble des $\phi$-types complets à paramètres dans $A$ qui sont consistants avec la théorie de $M$. C'est un espace topologique compact et séparé dont une base d'ouverts fermés est donnée par l'ensemble des $\phi$-formules sur $A$. On note $[\psi]$ l'ouvert fermé $\left\{p \in S_{\phi}(A): \psi \in p\right\}$ associé à la $\phi$-formule $\psi$.

\section{Structures Fines}

La chaîne des dérivées itérées d'un espace topologique $X$ est une chaine de fermés de $X$ définie inductivement en appelant $X^{\prime}$ l'ensemble des points d'accumulation de $X$ puis en posant

(1) $X^{\alpha+1}=\left(X^{\alpha}\right)^{\prime}$ pour un ordinal successeur $\alpha+1$,

(2) $X^{\lambda}=\bigcap_{\alpha<\lambda} X^{\alpha}$ pour un ordinal limite $\lambda$.

L'espace $X$ est clairsemé si cette chaîne aboutit au vide (c'est le cas en particulier si $X$ est un compact séparé dénombrable) : il y a un plus petit ordinal $\alpha$ tel que $X^{\alpha}$ soit vide. Si $X$ est compact, cet $\alpha$ a un prédécesseur $\beta$ que l'on appelle le rang de Cantor-Bendixson de $X$. Si $X$ est de plus séparé, $X^{\beta}$ est un ensemble fini. On appelle degré de Cantor-Bendixson de $X$ son cardinal.

Définition 1. Une théorie est fine si pour toute formule $\phi$ et tout ensemble fini $A$, l'espace topologique $S_{\phi}\left(d c l^{e q}(A)\right)$ est clairsemé.

Remarque. La finesse est une propriété de la théorie préservée par produits cartésiens finis : si la théorie de $M$ est fine, celle de $M^{n}$ l'est aussi. Elle est préservée par adjonction de la clôture imaginaire d'un nombre fini de paramètres au langage. Nous verrons qu'elle est aussi préservée par interprétation.

Pour incorporer les structures minimales, rajoutons :

Définition 2. Une structure $M$ est fine si pour tout uplet fini de paramètres a extrait de $M$ et pour toute formule $\phi(x, y)$ où l'arité de $x$ est égale à 1 , l'espace topologique $S_{\phi}\left(d c l^{e q}(a)\right)$ est clairsemé.

Remarques. - Si $A$ est un ensemble fini de paramètres et à moins qu'il y ait une infinité de constantes dans le langage, il n'y a qu'un nombre fini de $\phi$-formules sur $A$, donc $S_{\phi}(A)$ est fini.

- En général, $\left|S_{\phi}(\emptyset)\right|$ n'est pas égal à $\left|S_{\phi}(d c l(\emptyset))\right|$.

- Si $A$ est un ensemble fini, un $\phi$-type sur $d c l(A)$ n'a aucune raison d'être un $\phi$-type sur un ensemble fini.

- Une théorie est fine si et seulement si tous ses modèles le sont. Si une structure $\aleph_{0}$-saturée est fine, sa théorie l'est également. 
Exemples, contre exemples. (1) Une théorie est menue (small en anglais) si l'ensemble des $n$-types complets sans paramètres qui lui sont consistants est dénombrable pour tout entier $n$. Une théorie menue est fine : pour tout ensemble $A$ de taille finie $m$ et pour toute formule $\phi(x, y)$ où l'uplet de variables $x$ est de longeur $n$, on a

$$
\left|S_{\phi}\left(d c l^{e q}(A)|\leq| S_{n}(A)\right)\right| \leq\left|S_{n+m}(\emptyset)\right| \leq \aleph_{0}
$$

(2) Réciproquement, si dans une théorie $T$, l'ensemble $S_{\phi}\left(d c l^{e q}(A)\right)$ est dénombrable pour toute formule $\phi$ et tout partie finie $A$, il n'y a en général aucune raison que $T$ soit menue : il suffit de prendre dans un langage avec une infinité de prédicats binaires $E_{i}$ et de constantes, la théorie disant que les $E_{i}$ sont des relations d'équivalences ayant $2^{i}$ classes toutes infinies, chaque $E_{i+1}$ raffinant $E_{i}$ en coupant chacune de ses classes en deux, et chacune des classes de $E_{i}$ étant représentée par une constante du langage.

(3) Rappelons qu'une théorie est stable s'il existe un cardinal infini $\kappa$ tel que pour tout ensemble $A$ de paramètres, $|A| \leq \kappa$ implique $|S(A)| \leq \kappa$, ou encore s'il existe un modèle saturé $M$ de $T$ tel que $S_{\phi}(M)$ soit clairsemé pour toute formule $\phi(x, y)$. Une théorie stable $T$ est finie : si $A \subset B$, alors $C B\left(S_{\phi}(A)\right) \leq C B\left(S_{\phi}(B)\right)$. En particulier, si $S_{\phi}\left(M^{e q}\right)$ est clairsemé $S_{\phi}\left(d c l^{e q}(A)\right)$ l'est aussi pour toute partie finie $A$.

(4) Une structure $M$ est mince ${ }^{2}$ si pour tout uplet fini $a$ extrait de $M$, l'ensemble des 1-types complets sur $a$ est dénombrable : une structure mince (et en particulier une structure minimale, ou d-mininale au sens de [12, Poizat]) est fine.

(5) Le corps des réels n'est pas fin : dans le langage des corps ordonnés, si l'on considère la formule $\phi\left(x, y_{1}, y_{2}\right)$ qui dit " $y_{1}<x<y_{2}$ ", l'espace topologique $S_{\phi}(d c l(\emptyset))$ est à base dénombrable, mais son cardinal est $2^{\aleph_{0}}$. Rappelons que les corps réels clos sont dépendants : une théorie dépendante n'est en général pas fine. Remarquons au passage que pour une théorie, être dépendant n'est pas toujours préservé par ajout des paramètres de la clôture définissable du vide comme nouvelles constantes du langage.

(6) Une théorie supersimple n'est pas toujours fine. Les corps pseudo finis sont supersimples, mais ont une extension d'Artin-Schreier contrairement aux théories fines de corps (voir théorème 22).

\section{Le $\phi$-RANG De CANTOR}

Dans la structure $M$, soit $A$ un uplet fini de paramètres, $\phi(x, y)$ une formule et $F$ un $\phi$-ensemble sur $d c l^{e q}(A)$. Le $\phi$-rang de Cantor de $\phi$ sur $A$ est défini par l'induction suivante :

$R C_{\phi, A}(F) \geq 0$ si $F$ est consistant.

$R C_{\phi, A}(F) \geq \alpha+1$ s'il y a une infinité $X_{1}, X_{2}, \ldots$ de $\phi$-ensembles sur $d c l^{e q}(A)$ inclus dans $F$, deux à deux disjoints (de manière équivalente, dont l'intersection deux à deux a un rang strictement inférieur à $\alpha$ ), et ayant un rang de Cantor sur $A$ au moins égal à $\alpha$.

$R C_{\phi, A}(F) \geq \lambda$ pour un ordinal limite $\lambda$ si $R C_{\phi, A}(F) \geq \beta$ pour tout $\beta<\lambda$.

2. Le terme est suggéré par Poizat comme transcription de l'anglais weakly small 
Si $p$ est un $\phi$-type sur $d c l^{e q}(A)$, on définit $R C_{\phi, A}(p)$ par

$$
R C_{\phi, A}(p)=\min \{R C(\psi): \psi \in p\}
$$

Le $\phi$-rang de Cantor sur $A$ correspond bien sûr au rang de Cantor-Bendixson dans l'espace topologique $S_{\phi}\left(d c l^{e q}(A)\right)$ :

Lemme 3. Pour tout $p$ dans $S_{\phi}\left(d c l^{e q}(A)\right)$, l'ordinal $R C_{\phi, A}(p)$ est égal à $C B(p)$.

Démonstration. Par définition $C B(p)$ est le plus petit ordinal $\alpha$ tel que le dérivé $\left(S_{\phi}\left(d c l^{e q}(A)\right)^{\alpha}\right.$ contienne $p$. Si $C B(p)$ est nul, $p$ est isolé dans $S_{\phi}\left(d c l^{e q}(A)\right)$ par une $\phi$-formule donc $R C_{\phi, A}(p)=0$. Par induction, si $C B(p) \geq \alpha+1$, alors quelque soit la formule $\psi$ de $p,[\psi]^{\alpha+1}$ n'est pas vide. $[\psi]^{\alpha}$ possède une infinité de points $p_{1}, p_{2}, \ldots$ isolés par des $\phi$-formules sur $d c l^{e q}(A)$ dont on ne perd rien à supposer qu'elles sont deux-à-deux inconsistantes, donc $R C_{\phi, A}(\psi) \geq \alpha+1$ et $R C_{\phi, A}(p) \geq \alpha+1$. Réciproquement toujours par induction, si $R C_{\phi, A}(p) \geq \alpha+1$, soit $\psi$ une formule de $p$. On a $R C_{\phi, A}(\psi) \geq \alpha+1$ donc il existe une infinité de $\phi$-formules deux-à-deux inconsistantes de rang au moins $\alpha$ impliquées par $\psi$. En particulier $[\psi]^{\alpha}$ est infini par hypothèse d'induction. Il possède un point d'accumulation donc $[\psi]^{\alpha+1}$ est non vide, et ce pour toute formule $\psi$ de $p$. Donc $C B(p) \geq \alpha+1$.

Remarque. Dans le cas stable, les rang locaux sont finis par compacité. Ça n'est pas le cas ici. Rajouter l'exemple.

Le $\phi$-degré de Cantor de $F$ sur $A$ est le plus grand entier $d$ tel que $F$ contienne $d$ $\phi$-ensembles sur $d c l^{e q}(A)$ ayant un $\phi$-rang de Cantor maximal sur $A$. C'est aussi le nombre de types de $S_{\phi}\left(d c l^{e q}(A)\right)$ contenant $F$ ayant un $\phi$-rang de Cantor maximal sur $A$. Nous noterons $d C_{\phi, A}(F)$ ce degré.

Si $X$ est un ensemble $d c l^{e q}(A)$-définissable quelconque, on généralise la construction précédente en posant

(1) $R C_{\phi, A}(X)$ égal au maximum des rangs des $\phi$-types sur $d c l^{e q}(A)$ qui lui sont consistants (c'est-à-dire au rang de Cantor-Bendixson de $\left.S_{\phi}^{X}\left(d c l^{e q}(A)\right)\right)$;

(2) $d C_{\phi, A}(X)$ égal au degré de Cantor-Bendixson de $S_{\phi}^{X}\left(d c l^{e q}(A)\right)$.

Si $A$ est vide, nous écrirons $R C_{\phi}(X)$ et $d C_{\phi}(X)$ plutôt que $R C_{\phi, \emptyset}(X)$ et $d C_{\phi, \emptyset}(X)$.

Lemme 4. A est un ensemble de paramètres, $\phi$ une formule, $X$ et $Y$ sont deux sous-ensembles définissables sur $d l^{e q}(A)$ dans une structure quelconque. On a les formules usuelles:

(1) $R C_{\phi, A}(X \cup Y)=\max \left\{R C_{\phi, A}(X), R C_{\phi, A}(Y)\right\}$.

(2) $d C_{\phi, A}(X \cup Y)+d C_{\phi, A}(X \cap Y)=d C_{\phi, A}(X)+d C_{\phi, A}(Y)$.

Soit $\phi(x, y)$ une formule, et soient $X$ et $Y$ deux ensembles définissables de même arité que l'uplet $x$. Si $g$ est une application définissable de $X$ vers $Y$, on note $g^{-1} \phi$ la formule $\phi(g(x), y)$ c'est-à-dire la formule $(\exists z)(z=g(x) \wedge g(z, y))$.

Lemme 5. Si $X$ et $Y$ et $g$ sont tous trois $d c^{e q}(A)$-définissables, alors,

(1) Sig est surjective, $R C_{g^{-1} \phi, A}(X) \geq R C_{\phi, A}(Y)$

(2) Sig est à fibres finies, $R C_{g^{-1} \phi, A}(X) \leq R C_{\phi, A}(Y)$ 
(3) Sig est surjective et si ses fibres n'ont pas plus de $n$ éléments, alors $R_{g^{-1} \phi, A}(X)$ et $R C_{\phi, A}(Y)$ sont égaux, et

$$
d C_{\phi, A}(Y) \leq d C_{g^{-1} \phi, A}(X) \leq n \cdot d C_{\phi, A}(Y)
$$

Démonstration. Commençons par rajouter les paramètres de $A$ au langage. On note $t p_{\phi}(x)$ le $\phi$-type de $x$ sur $d c l^{e q}(\emptyset)$. Comme dans [8, Remark 1.9], il suffit de remarquer que l'application $g$ de $X$ dans $Y$ induit une application $G$ de $S_{g^{-1} \phi}^{X}\left(d c l^{e q}(\emptyset)\right)$ dans $S_{\phi}^{Y}\left(d c l^{e q}(\emptyset)\right)$ en posant $G\left(t p_{g^{-1} \phi}(x)\right)$ égal à $t p_{\phi}(g(x))$. Cette application est continue et ouverte. Si $g$ est surjective, $G$ l'est aussi. La taille des fibres de $g$ majore celle des fibres de $G$. On conclut avec le lemme 3 et [8, Lemma 1.10].

Proposition 6. $M$ est une structure fine, $A$ un sous-ensemble fini de paramètres et $E$ une relation d'équivalence définissable sur $d c l(A)^{e q}$. L'union disjointe de $M$ et $M / E$ est fine. Le langage considéré est celui de $M$ augmenté d'un symbol de de fonction $\pi_{E}$ pour la surjection canonique de $M$ dans $M / E$ et d'un prédicat unaire interprétant $M / E$.

Démonstration. Dans ce nouveau langage, l'ensemble définissable $M$ est encore fin. D'après le lemme 5.1, l'ensemble $M / E$ l'est aussi. On conclut avec le lemme 4.1.

Définition 7. Soit $M$ une strucure, et $\mathfrak{C}$ une extension élémentaire saturée de $M$. Si $a$ est un paramètre dans $\mathfrak{C}$, et $\phi(x, y)$ une formule, on notera $\bar{\phi}(x, a)$ la disjonction des formules $\phi(x, b)$ où $b$ parcourt l'ensemble des conjugués de $a$ sous l'action de Aut $(\mathfrak{C})$. On notera $\stackrel{\circ}{\phi}(x, a)$ la conjonction de ces formules. Si $X$ est le sous-ensemble de $M$ défini par $\phi(x, a)$, on note $\bar{X}$ pour l'ensemble réalisant $\bar{\phi}(x, a)$, et $\stackrel{\circ}{X}$ pour celui réalisant $\stackrel{\circ}{\phi}(x, a)$.

Si $X$ est le singleton réduit à $a$, on préferera écrire $\bar{a}$ à $\overline{\{a\}}$.

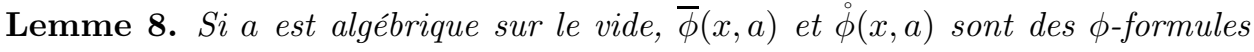
sur la clôture imaginaire du vide.

Démonstration. Soit $\psi$ une formule algébrique minimale sur le vide vérifiée par $a$, et $E$ la relation d'équivalence $\emptyset$-définissable correspondant à la partition $\psi, \neg \psi$. L'ensemble $\bar{a}$ n'est autre que la classe $a_{E}$ de $a$ pour $E$, et $\bar{\phi}(x, a)$ est équivalente à $\phi\left(x, a_{E}\right)$. D'autre part, à équivalence logique près on a

$$
\dot{\phi}(x, a)=\neg \neg \bigwedge_{b \in \bar{a}} \phi(x, b)=\neg \bigvee_{b \in \bar{a}} \neg \phi(x, b)=\neg(\overline{(\neg \phi)}(x, a))=\neg\left((\neg \phi)\left(x, a_{E}\right)\right)
$$

Remarquons enfin que rajouter un nombre fini de paramètres algébriques au langage n'affecte aucun $\phi$-rang :

Lemme 9. Soit $X$ un ensemble définissable sans paramètres, et a un élément algébrique de degré $n$ sur le vide. Alors, pour toute formule $\phi$,

(1) $R C_{\phi, \bar{a}}(X)=R C_{\phi, \emptyset}(X)$

(2) $d C_{\phi, \emptyset}(X) \leq d C_{\phi, \bar{a}}(X) \leq n ! \cdot d C_{\phi, \emptyset}(X)$ 
Démonstration. Nous suivons encore [8, Remark 1.9]. On ne perd rien à supposer que $X$ est la structure ambiante. On considère l'application restriction de $S_{\phi}\left(d c l^{e q}(\bar{a})\right)$ dans $S_{\phi}\left(d c l^{e q}(\emptyset)\right)$. C'est une application continue et surjective. Montrons que ses fibres n'ont pas plus de $n$ ! éléments. Soit $a_{1}, \ldots, a_{n}$ la liste des conjugués de $a$ sous l'action de $\operatorname{Aut}(\mathfrak{C})$. Si $x$ et $y$ ont le même $\phi$-type sur $d c^{l e q}(\emptyset)$ et si $f_{1}\left(x, a_{1}, \ldots, a_{n}\right)$ est dans le $\phi$-type de $x$ sur $d c l^{e q}(\bar{a})$, alors $\bar{f}_{1}\left(x, a_{1}, \ldots, a_{n}\right)$ est dans le $\phi$-type de $x$ (et de $y$ ) sur le $d c l^{e q}(\emptyset)$ d'après le lemme 8 . On en déduit que $f_{1}\left(x, a_{\sigma(1)}, \ldots, a_{\sigma(n)}\right)$ est dans le $\phi$-type de $y$ sur $d c l^{e q}(\bar{a})$ pour une certaine permutation $\sigma$ des coordonnées $\{1, \ldots, n\}$. Si l'on itère le raisonnement avec une deuxième formule $f_{2}$, en considérant la conjonction $f_{1} \wedge f_{2}$, on voit que la permutation $\sigma$ ne dépend pas de la formule choisie, d'où les $n$ ! choix. On conclut avec le lemme 3 et [8, Lemma 1.10].

Si $X$ est un ensemble sur définissable sur un ensemble fini $a$, on appelle $\phi$-rang de Cantor local de $X$ sur $a$ son $\phi$-rang de Cantor sur n'importe quel ensemble fini $b$ permettant de définir $X$ et tel que $b \subset \operatorname{acl}(a)$ et $a \subset \operatorname{acl}(b)$.

\section{GÉnÉRAlités sur LES GROUPES Fins}

Dans un groupe $G$, pour une formule $\phi(x, y)$, on notera $z \phi(x, y)$ ou simplement $z \phi$ la formule $\phi\left(z^{-1} \cdot x, y\right)$ où $z$ est une nouvelle variable de paramètres d'arité 1 . De même, les formules $\phi z(x, y)$ et $z \phi t(x, y)$ voudront dire $\phi\left(x \cdot z^{-1}, y\right)$ et $\phi\left(z^{-1} \cdot x \cdot t^{-1}, y\right)$ respectivement.

Corollaire 10. Soit $h$ un homomorphisme définissable d'un groupe fin $G$, dont le noyau ait au plus $n$ éléments. S'il existe un uplet fini $b$ de paramètres dans $G$ tels que les images itérées de $h$ sont uniformément définissables sur $d c l^{\text {eq }}(b)$, alors $h(G)$ est d'indice au plus $n$ dans $G$.

Démonstration. Sinon, on peut trouver un uplet fini $a$ avec lequel on définit $n+1$ classes de $G$ modulo $h(G)$. Soit $\psi(x, y)$ la formule permettant de définir les images itérées de $h$. On considère la formule $\phi(x, y)=y \psi(x, y)$. Le $\phi$-degré de $G$ sur $\{a, b\}$ est au moins $(n+1) \cdot d C_{\phi, a, b}(h(G))$, ce qui contredit le lemme 5 .

En fait, sans hypothèse sur le noyau mais en supposant la théorie fine, on a la chose suivante :

Proposition 11. Soit un groupe $G$ dont la théorie est fine. Soit un uplet fini b de paramètres de $G$, un homomorphisme définissable $h$ du groupe $G$ dont les images itérées soient uniformément définissables sur $d_{c l} l^{e q}(b)$. Alors la chaîne des images itérées stationne. En particulier, il y a un entier $n$ tel que $G$ se décompose en le produit $h^{n}(G) \cdot h^{-n}(\{1\})$.

Démonstration. Si la chaîne $h(G), h^{2}(G), \ldots$ décroît strictement, soit $A(x)$ l'arbre binaire suivant

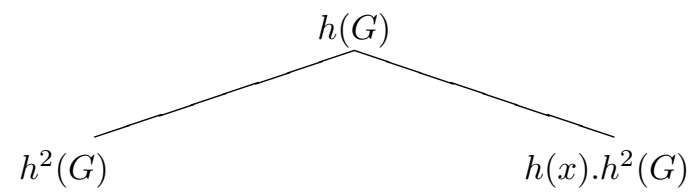



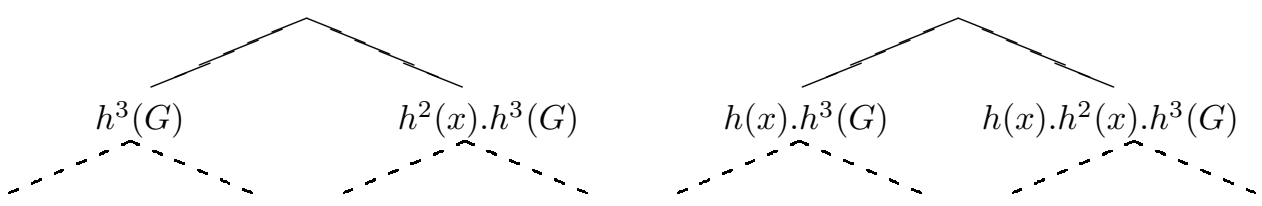

Appelons $\pi(x)$ le type partiel $\left\{x \notin h^{-n}\left(h^{n+1}(G)\right), n \geq 1\right\}$. Les formules de $\pi$ sont uniformément définissables par une formule $\phi$ disons. La suite $h^{-1} h^{2}(G), h^{-2} h^{3}(G), \ldots$ est croissante, et chaque $G \backslash h^{-n} h^{n+1}(G)$ est non vide par hypothèse, donc $\pi$ est finiment consistant. Soit $a$ une réalisation de $\pi$ dans un modèle saturé. L'arbre $A(a)$ a $2^{\aleph_{0}}$ branches consistantes, donc $S_{\phi}\left(d c l^{e q}(a, b)\right)$ est de cardinal $2^{\aleph_{0}}$, une contradiction. Il y a donc un entier $n$ tel que $h^{n}(G)=h^{2 n}(G)$, et on en déduit que $G=h^{n}(G) \cdot h^{-n}(\{1\})$.

Lemme 12. Soit $G$ un groupe fin, $\phi(x, y)$ une formule, et $H_{2} \leq H_{1}$ deux sousgroupes de $G$ qui soient définis par des $\phi$-ensembles sur $d c^{e q}(\emptyset)$.

(1) Si $H_{2} \cap a c l(\emptyset)$ est un sous-groupe propre de $H_{1} \cap a c l(\emptyset)$, alors soit $R C_{z \phi}\left(H_{2}\right)<$ $R C_{z \phi}\left(H_{1}\right)$, soit $d C_{z \phi}\left(H_{2}\right)<d C_{z \phi}\left(H_{1}\right)$.

(2) Si $H_{1}$ et $H_{2}$ ont même $z \phi$-rang de Cantor sur le vide, alors $H_{2} \cap$ acl $(\emptyset)$ est d'indice fini dans $H_{1} \cap \operatorname{acl}(\emptyset)$.

Démonstration. Soit $b$ un élément de $\operatorname{acl}(\emptyset)$ dans $H_{1} \backslash H_{2}$. L'ensemble $\overline{b . H_{2}}$ est un $z \phi$-définissable sur $d c l^{e q}(\emptyset)$ d'après le lemme 9 et disjoint de $H_{2}$ donc soit me $z \phi$ rang de Cantor de $H_{2}$ n'est pas maximal, soit son degré ne l'est pas. Supposons $H_{1}$ et $H_{2}$ de même $z \phi$-rang de Cantor sur le vide. D'après les lemmes 9,5 et 4 , on a

$$
R C_{z \phi}\left(H_{2}\right)=R C_{z \phi, b}\left(H_{2}\right)=R C_{z \phi, b}\left(b H_{2}\right)=R C_{z \phi, b}\left(\overline{b . H_{2}}\right)=R C_{z \phi}\left(\overline{b . H_{2}}\right)
$$

Le $z \phi$-rang de Cantor de $\overline{b . H_{2}}$ sur le vide est maximal dans $H_{1}$, et il ne peut y avoir qu'un nombre fini de choix pour $\overline{b . H_{2}}$, et donc pour $b . H_{2}$.

Théorème 13 (Condition de chaîne uniforme et locale). Dans un groupe fin, pour tout uplet fini $b$ de paramètres de $G$, la trace sur acl(b) d'une chaîne décroissante de sous-groupes définis par des $\phi$-formules sur acl(b) stationne après un nombre fini d'étapes.

Démonstration. On ne perd rien à rajouter les éléments de $b$ au langage. Soit $G_{1} \geq G_{2} \geq \ldots$ une chaîne décroissante de sous-groupes uniformément acl( $\left.\emptyset\right)$ définissables. Appelons $\phi(x, y)$ la formule les définissant, et $\psi(x, y, z)$ la formule $z \phi(x, y)$. Après un nombre fini d'étapes, le $\psi$-rang de Cantor local des $G_{i}$ stationne d'après le lemme 9 , donc on ne perd rien à supposer qu'il est constant dès le départ. L'indice de $G_{i} \cap \operatorname{acl}(\emptyset)$ dans $G_{1} \cap \operatorname{acl}(\emptyset)$ est fini pour tout $i$ d'après les lemmes 12 et 9. On peut ajouter un nombre fini de paramètres algébriques au langage et supposer que $G_{1}$ est définissable sans paramètres. Pour tout $i$, l'ensemble $\dot{G}_{i} \cap \operatorname{acl}(\emptyset)$ est un $\phi$-ensemble définissable sur $d c l^{e q}(\emptyset)$. L'intersection sur $i \operatorname{des} \stackrel{G}{i}_{i} \cap a c l(\emptyset)$ est l'intersection d'un nombre fini d'entre eux d'après le lemme $12:$ c'est un sous-groupe de $G_{0} \cap \operatorname{acl}(\emptyset)$ d'indice fini, qui est inclus dans chacun des $G_{i}$. Les suites des indices $\left[G_{0} \cap \operatorname{acl}(\emptyset): G_{i} \cap \operatorname{acl}(\emptyset)\right]$ est donc bornée, ce qui majore la longueur de la châne $G_{1} \cap \operatorname{acl}(\emptyset) \geq G_{2} \cap \operatorname{acl}(\emptyset) \geq \ldots$

Illustrons ce théorème avec plusieurs conséquences : 
Corollaire 14. $G$ est un groupe mince $G$ et $\Gamma$ une clôture algébrique finiment engendrée dans $G$. Pour toute partie $A$ de $\Gamma$ il y a un ensemble fini $B \subset A$ tel que

$$
C_{\Gamma}(A)=\bigcap_{a \in B} C_{\Gamma}(a)
$$

Corollaire 15. Un groupe infini dont le centre est d'indice infini, et n'ayant qu'une seule classe de conjugaison non-centrale n'est pas fin.

Remarque. Cela généralise les cas stable [10, Théorème 3.10] et mince [8].

Démonstration. Le groupe n'a pas de second centre. Quitte à quotienter, on peut supposer que le centre est réduit au neutre. S'il y avait une autre involution que le neutre, tous les éléments seraient des involutions et le groupe serait abélien. A part le neutre, tout élément $g$ est donc conjugué à $g^{-1}$ par un élément $h$ non trivial. $h$ est conjugué à $h^{2}$ : il est égal à $k^{-1} h k$ pour un certain $k$ de $G$. Soit $\delta$ la clôture définissable de $h$ et de $k$. Puisque $g$ est dans $C\left(h^{k}\right)$ et que $g h \neq h g$, l'élément $h$ est dans $(C(C(h)) \cap \delta) \backslash\left(C\left(C\left(h^{k}\right)\right) \cap \delta\right)$. On en déduit que la chaîne

$$
C(C(h)) \cap \delta>C\left(C\left(h^{k}\right)\right) \cap \delta>C\left(C\left(h^{k^{2}}\right)\right) \cap \delta>\cdots
$$

est infinie, une contradiction avec la condition de chaîne 13.

Corollaire 16. Dans un groupe fin $G$, soit $\Gamma$ une clôture algébrique finiment engendrée et $A_{1}, A_{2}, \ldots$ une suite de parties de $\Gamma$. Toute chaîne croissante de centralisateurs de la forme $C\left(A_{1}\right) \leq C\left(A_{2}\right) \leq \cdots$ stationne après un nombre fini d'étapes.

Démonstration. Supposons que la chaîne $C\left(A_{1}\right)<C\left(A_{2}\right)<\cdots$ soit strictement croissante. Il existe une suite $a_{1}, a_{2}, \ldots$ dans $A_{1} \times A_{2} \times \ldots$ et une autre $x_{1}, x_{2}, \ldots$ dans $G^{\omega}$ telles que $x_{i}$ et $A_{i}$ commutent, mais que $x_{i}$ et $a_{i+1}$ ne commutent pas, et ce quel que soit $i$. Ces deux suites témoignent du fait que la chaîne $C_{\Gamma}\left(C\left(A_{1}\right)\right)>$ $C_{\Gamma}\left(C\left(A_{2}\right)\right)>\cdots$ est strictement décroissante, contredisant le théorème 13.

Définition 17. Soit $X$ un ensemble définissable d'un groupe fin $G$, soit $\phi(x, y)$ une formule, et $\Gamma$ la clôture algébrique d'un uplet fini $g$ de $G$. On définit le $\phi$-presque stabilisateur local de $X$ dans $\Gamma$ par

$$
\operatorname{Stab}_{\Gamma}^{\phi}(X)=\left\{h \in \Gamma: R C_{z \phi, h, g}(h X \Delta X)<R C_{z \phi, h, g}(X)\right\}
$$

Si $\delta$ est un sous groupe de $\Gamma$, on pose $\operatorname{Stab}_{\delta}^{\phi}(X)=\operatorname{Stab}_{\Gamma}^{\phi}(X) \cap \delta$.

Remarque. Pour un ensemble définissable $X$ du groupe fin $G$, on n'a pas nécessairement l'égalité des rangs $R C_{z \phi}(X), R C_{\phi z}(X)$ et $R C_{z \phi s}(X)$. Cependant, si $g$ est une bijection definissable de $G$ dans $G$, alors, pour toute formule $\phi$, on a $R C_{g^{-1} \phi}(G)=R C_{\phi}(G)$. Cela vaut en particulier pour l'opération inverse ${ }^{-1}$, et, pour un ensemble définissable $X$, être de $z \phi$-rang maximal, c'est être de $\phi z$-rang maximal, ou encore de $z \phi s$-rang maximal.

Corollaire 18. Si $X$ n'est pas vide et si $(\exists y) \phi(x, y)$ est consistante, il existe un élément $g$ de $G$ tel que pour toute clôture algébrique finiment engendrée $\Gamma$ contenant $g$ l'ensemble $S_{t a b}^{\phi}(X)$ soit un sous-groupe de $\Gamma$. Si de plus $X$ est invariant par conjugaison par $\Gamma$ alors $\operatorname{Stab}_{\Gamma}^{\phi}(X)$ est distingué dans $\Gamma$. 
Démonstration. Soient $a$ et $g$ dans $G$ vérifiant $\phi(a, g)$ et soit $h$ dans $X$. On a $\left(a h^{-1}\right) h=a$ donc $X$ et $\phi\left(a h^{-1} x, g\right)$ sont consistants et $R C_{z \phi, g}(X) \geq 0$ d'où $1 \in$ $S t a b_{\Gamma}^{\phi}(X)$ dès que $\Gamma$ contient $g$. Soient $a$ et $b$ dans $S t a b_{\Gamma}^{\phi}(X)$. D'après le lemme 5 les ensembles $X, a X$ et $b X$ ont les mêmes $z \phi$-types de rang maximal calculés sur les paramètres $g, a, b$ donc

$$
R C_{z \phi, g, a, b}(a X \Delta b X)<R C_{z \phi, g}(X)
$$

Puisque le $z \phi$-rang est préservé par ajout de paramètres algébriques, et par translation par un élément de $\operatorname{acl}(\emptyset)$, on a

$$
R C_{z \phi, g, a, b}(a X \Delta b X)=R C_{z \phi, g, a, b}\left(b^{-1} a X \Delta X\right)=R C_{z \phi, g, b^{-1} a}\left(b^{-1} a X \Delta X\right)
$$

donc $b^{-1} a$ est dans $\operatorname{Stab}_{\Gamma}^{\phi}(X)$.

Dans un groupe stable $G$, on peut définir le $\phi$-stabilisateur $S t a b^{\phi}(X)$ d'un ensemble définissable $X$, et ce $\operatorname{Stab}^{\phi}(X)$ est d'indice fini pour peu que $X$ soit générique. Dans un groupe menu, pour toute clôture définissable finiment engendrée $\delta$, il existe une notion de presque stabilisateur local $\operatorname{Stab}_{\delta}(X)$ d'un ensemble $\delta$-définissable $X$, tel que $\operatorname{Stab}_{\delta}(X)$ soit d'indice fini dans $\delta$ dès que $X$ est de rang de Cantor maximum $\operatorname{sur} \delta[8]$. Dans un groupe fin :

Proposition 19. Soit $G$ un groupe fin, $g$ un uplet fini de $G, \phi(x, y)$ une formule, et $X$ un sous-ensemble $g$-définissable de $G$. Si $\delta$ est un sous-groupe de $d c l(g)$ et si $X$ est de $z \phi$-rang de Cantor maximal sur $g$, alors $\operatorname{Stab}_{\delta}^{\phi}(X)$ est d'indice fini dans $\delta$.

Démonstration. Soient $m$ et $l$ les $z \phi$-degrés de $G$, et $X$ sur $g$. Il y a $m z \phi$-types de rang sur $g$ maximal dans $G$ que nous appelons ses $z \phi$-types génériques. Donc, pour un translaté de $X$ par un élément de $\delta$, il y a au plus $C_{m}^{l}$ choix possibles pour ses $z \phi$-types génériques. Si l'on choisit $C_{m}^{l}+1$ translatés de $X$, on s'assure que au moins deux d'entre eux auront les mêmes $z \phi$-types génériques.

\section{CoRps FIns}

Théorème 20. Un corps infini dont la théorie est fine n'a pas de sous-groupe définissable propre d'indice fini, ni additif, ni multiplicatif. Qui plus est, un corps fin n'a pas de sous-groupe définissable additif propre d'indice fini.

Démonstration. Soit $K$ ce corps fin. Si $H$ est un sous-groupe additif définissable de $K$ d'indice fini dans $K^{+}$. Supposons tout d'abord que $K$ ne soit pas localement fini. On considère alors une clôture algébrique infinie $\Gamma$ finiment engendrée qui contient un représentant de chaque classe de $H$. La trace d'un translaté multiplicatif de $H$ dans $\Gamma$ est aussi d'indice fini dans $\Gamma$. La trace sur $\Gamma$ de l'intersection des $a H$ quand $a$ parcourt $\Gamma$ stationne. C'est un idéal de $\Gamma$ d'indice fini qui est donc $\Gamma$ tout entier. On vient de montrer $\Gamma \subset H$. Comme ceci est valable pour toute clôture $\Gamma$ finiment engendrée, on a $H=K$.

Si $K$ est localement fini, il est commutatif et égal à $a c l(\emptyset)$. D'après la condition de chaîne 13 , l'intersection des $a H$ quand $a$ parcourt $K$ stationne et $H=K$.

Soit maintenant $M$ un sous-groupe de $K^{\times}$d'indice fini. Si la théorie de $K$ est finie, par compacité il y a des éléments d'ordre arbitrairement grand et on peut supposer que $K$ n'est pas localement fini quitte à le remplacer par une extension élémentaire. Soit $\delta$ un sous-corps infini finiment engendré contenant un représentant 
de chaque classe de $M$. Si $\phi$ désigne la formule définissant $M$, d'après les lemmes 4 et 5 l'ensemble $M$ est de $z \phi$-rang maximal sur $\delta$, donc son $\phi$-presque stabilisateur additif dans $\delta$ est d'indice fini dans $\delta$ d'après la proposition 19, de même que le $\phi$-presque stabilisateur de chacune de ses classes à gauche. L'intersection de ces stabilisateurs est un idéal à gauche de $\delta$ d'indice fini, c'est-à-dire $\delta$ tout entier. Comme dans [12, Poizat] nous venons de montrer que $1+a M \simeq_{z \phi} a M$ pour toute classe $a M$, où $\simeq_{z \phi}$ désigne l'égalité à petit $z \phi$-rang de Cantor sur $\delta$ près. Pour tout classe $a M$ et tout $x$ dans $a M$ sauf un ensemble de petit $z \phi$-rang de Cantor local, $1+x \in a M$, donc $x^{-1}+1 \in M$. Le complémentaire de $M$ est de petit $z \phi$-rang de Cantor. On en déduit que $M=K^{\times}$.

Si $K$ est un corps, on note $K^{a l g}$ sa clôture algébrique. Nous avons besoin du résultat suivant sur les groupes algébriques pour montrer le théorème 22 .

Fait 21. [4, Corollaire 2.6]. $K$ est un corps parfait, $n \geq 1$ un entier naturel, et $G\left(K^{a l g}\right)$ un sous-groupe algébrique de $\left(K^{a l g},+\right)^{n}$ défini sur $K$, fermé, connexe et de dimension 1 . Alors $G\left(K^{\text {alg }}\right)$ est isomorphe à $\left(K^{\text {alg }},+\right)$ au-dessus de $K$.

Théorème 22. Un corps infini dont la théorie est fine n'a pas d'extension d'ArtinSchreier.

Démonstration. Soit $K$ ce corps, et $K^{\text {par }}$ le corps $\bigcap_{n \geq 1} K^{p^{n}}$, qui est parfait et que l'on peut supposer infini par compacité quitte à remplacer $K$ par une extension élémentaire saturée. Soit $k$ une clôture algébrique infinie finiment engendrée de $K^{p a r}$. Le corps $k$ est lui aussi parfait. On considère l'application d'Artin-Schreier $\phi$, qui à un $x$ de $K$ associe $x^{p}-x$. D'après la condition de chaîne uniforme et locale 13, le groupe $I$ défini par $\cap_{a \in k} \times a \phi(K) \cap k$ est égal à une intersection finie de la forme $\bigcap_{i=1}^{n} a_{i} \phi(K) \cap k$ où $a_{1}, \ldots, a_{n}$ sont dans $k^{\times}$. Nous reprenons ensuite un argument de Scanlon [13,4] : considérons le groupe algébrique linéaire $G\left(k^{\text {alg }}\right)$ de $\left(\left(k^{a l g}\right)^{+}\right)^{n+1}$ défini par les équations polynômiales $\bigwedge_{i=1}^{n} a_{i}\left(X_{i}^{p}-X_{i}\right)-X_{n+1}$. Etant l'intersection de $n$ hypersurfaces, $G\left(k^{\text {alg }}\right)$ est de dimension au moins 1. Puisque $k$ est parfait, d'après le fait 21 , la composante connexe de l'unité de $G\left(k^{a l g}\right)$ est isomorphe à $\left(k^{\text {alg }}\right)^{+}$au-dessus de $k$. Cet isomorphisme envoie $G(k)$ sur $k^{+}$. En particulier, $G(k)$ est infini. La projection de $G(k)$ sur la dernière coordonnée étant à fibres finies, on peut trouver un élément $a$ non nul dans $\cap_{a \in k \times} a \phi(K) \cap k$. Mais $\cap_{a \in k^{\times}} a \phi(K) \cap k$ est un idéal de $k$, donc égal à $k$ tout entier, et $k \subset \phi(K)$. Ceci étant valable pour toute clôture algébrique finiment engendrée dans $K^{\text {par }}$, on a $K^{p a r} \subset \phi(K)$. Par compacité, il existe un $n$ tel que $K^{p^{n}} \subset \phi(K)$. On en déduit sans peine que $\phi\left(K^{p^{n}}\right)=K^{p^{n}}$, puis que $\phi(K)=K$.

Corollaire 23. Soit $K$ un corps infini de caractéristique $p$ dont la théorie est fine, et $L$ une extension séparable de $K$. Le degré de $L / K$ n'est pas divisible par $p$.

Démonstration. S'il y a une extension algégrique séparable de $K$ dont le degré sur $K$ divise $p$, il y a aussi une extension galoisienne $M$ dont le degré sur $K$ divise $p$. Son groupe de Galois a un sous-groupe d'ordre $p$, dont on note $K_{1}$ le corps des invariants. L'extension $L / K_{1}$ est d'Artin-Schreier. Mais $K_{1}$ étant une extension algébrique de $K$ est interprétable dans une puissance cartésienne de $K$ : sa théorie est donc fine. 
Théorème 24. Soit un corps fin de caractéristique positive p. Pour tout ulet fini $b$ du corps, le sous-corps acl(b) est de dimension finie sur son centre.

Démonstration. Soit $K$ ce corps, $a$ un élément hors du centre, $b$ un élément ne commutant pas avec $a$, et $h$ l'application qui à un $x$ de $K$ associe $a^{-1} x a-x$. Puisque les corps finis sont commutatifs, $K$ n'est pas localement fini. Soit $k$ une clôture algébrique infinie finiment engendrée de $K$ contenant $a$ et $b$. D'après la condition de chaîne, et parce que les puissances itérées de $h$ sont uniformément $k$-définissables, les chaînes $k \cap h^{n}(K)$ et $k \cap h^{-n}(\{0\})$ stationnent toutes les deux à partir d'un certain rang $m$. On montre alors sans peine que

$$
k \subset h^{-m}(\{0\})+h^{m}(K)
$$

D'après le corollaire 16 , la chaîne de centralisateurs $C\left(a^{p^{i}}\right)$ stationne, donc celles des noyaux itérés de $h$ également : quitte à augmenter $m$, on peut supposer que $h^{-m}(\{0\})$ et $h^{m}(K)$ sont en somme directe. Puisque la suite des dimensions des noyaux itérés d'un endomorphisme s'essouffle, $h^{-m}(\{0\})$ est de dimension finie (au plus $m$ ) sur $C(a)$. On a donc

$$
k=h^{-m}(\{0\}) \cap k \oplus h^{m}(K) \cap k
$$

Soit $I$ l'intersection $\bigcap_{a \in k^{\times}} a h^{m}(K) \cap k$. D'après le théorème 13 , l'ensemble $I$ est une intersection finie de sous-espaces vectoriels sur le corps $C_{k}(a)$ qui sont chacun de codimension finie sur $C_{k}(a)$. Mais c'est aussi un idéal à gauche qui ne contient pas 1. Il est donc nul, ce qui montre que la dimension de $k$ sur $C_{k}(a)$ est finie. Comme c'est valable pour n'importe quel $a$ de $k$ et que le centre de $k$ est l'intersection d'un nombre fini de centralisateurs par $13, k$ est de dimension finie sur son centre.

[6] montre qu'un corps stable de caractéristique positive est de dimension finie sur son centre. On peut préciser ce résultat :

Corollaire 25. Un corps fin de caractéristique positive sans propriété d'indépendance est de dimension finie sur son centre.

Définition 26 (Shelah). Dans une théorie $T$, une formule $\phi(x, y)$ à la propriété d'indépendance s'il y a deux suites $\left(a_{i}\right)_{i \in \mathbb{N}}$ et $\left(b_{I}\right)_{I \subset \mathbb{N}}$ telles que $\phi\left(a_{i}, b_{I}\right)$ soit valide dans $T$ si et seulement si $i \in I$. Une théorie est dépendante si aucune formule n'a la propriété d'indépendance. Une structure est dépendante si sa théorie l'est.

Fait 27 (Baldwin-Saxl). Dans une groupe dépendant, soit $H_{1}, H_{2}, H_{3} \ldots$ une famille de sous-groupes uniformément définissables. Il existe un entier naturel $N$ tel que toute intersection d'un nombre fini de groupes $H_{i}$ soit égale à l'intersection d'au plus $N$ d'entre eux.

Démonstration du corollaire 25. Il suffit de remarquer que puisque le corps est dépendant, dans la démonstration précédente toute les intersections de groupes uniformément définissables sont des intersections bornées d'après le fait 27. En particulier, il existe un entier $N$ tel que pour tout uplet $b$ fini, la dimension de $a c l(b)$ sur son centre soit au plus $N$. On en déduit que le corps est de dimension au plus $N$ sur son centre. 


\section{RÉFÉRENCES}

[1] Chantal Berline and Daniel Lascar, Superstable groups, Annals of Pure and Applied Logic 30, 1-43, 1986.

[2] Gregory Cherlin et Saharon Shelah, Superstable fields and groups, Annals of Mathematical Logic 18, 3, 227-270, 1980.

[3] Ehud Hrushovski, Contributions to stable model theory, PhD, Berkeley, 1986.

[4] Itay Kaplan, Thomas Scanlon et Frank O. Wagner Artin-Schreier extensions in dependent and simple fields, à paraître.

[5] Cédric Milliet, Small Skew fields, Mathematical Logic Quarterly 53, 86-90, 1, 2007.

[6] Cédric Milliet, Stable division rings, The Journal of Symbolic Logic 76, 1, 348-352, mars 2011.

[7] Cédric Milliet, On enveloping type-definable structures, The Journal of Symbolic Logic 76, 3, 1023-1034, septembre 2011.

[8] Cédric Milliet, On properties of (wealkly) small groups, à paraître, The Journal of Symbolic Logic.

[9] Cédric Milliet, Fields and rings with few types, preprint.

[10] Bruno Poizat, Groupes Stables, Nur Al-Mantiq Wal-Ma'rifah, 1987.

[11] Bruno Poizat et Frank O. Wagner, Sous-groupes périodiques d'un groupe stable, The Journal of Symbolic Logic, 58, 2, 385-400, juin 1993.

[12] Bruno Poizat, Quelques tentatives de définir une notion générale de groupes et de corps de dimension un et de déterminer leurs propriétés algébriques, Confluentes Mathematici, à paraître, 2009.

[13] Thomas Scanlon, Infinite stable fields are Artin-Schreier closed, non publié, 1999.

[14] Frank O. Wagner, Small stable groups and generics, The Journal of Symbolic Logic 56, 3, 1036-1037, septembre 1991.

[15] F. O. Wagner, Quasi-endomorphisms in small stable groups, The Journal of Symbolic Logic 58, 1044-1051, 1993.

[16] Frank O. Wagner, Small fields, The Journal of Symbolic Logic 63, 3, 995-1002, 1998.

Université Galatasaray

Faculté de Sciences et de Lettres

Département de Mathématiques

ÇIRAĞAN CADDESI N³6

34357 Ortaköy, Istamboul, Turquie

E-mail address: milliet@math.univ-lyon1.fr 